See discussions, stats, and author profiles for this publication at: https://www.researchgate.net/publication/336264321

\title{
The relationship between ICD-11 PTSD, complex PTSD and dissociative experiences
}

Article in Journal of Trauma \& Dissociation · October 2019

Dol: 10.1080/15299732.2019.1675113

CITATIONS

10

5 authors, including:

Philip Hyland

13 National University of Ireland, Maynooth

216 PUBLICATIONS 2,950 CITATIONS

SEE PROFILE

Marylene Cloitre

National Center for PTSD

219 PUBLICATIONS 12,615 CITATIONS

SEE PROFILE
READS

1,004

Some of the authors of this publication are also working on these related projects:

COVID-19 Psychological Research Consortium study (COVID-19 PRCS) View project

Predictors of treatment outcome for PTSD versus Complex PTSD View project

Mark Shevlin

Ulster University

416 PUBLICATIONS 9,925 CITATIONS

SEE PROFILE

Thanos Karatzias

Edinburgh Napier University

181 PUBLICATIONS 2,864 CITATIONS

SEE PROFILE 


\section{The relationship between ICD-11 PTSD, complex PTSD and dissociative experiences}

\section{Philip Hyland, Mark Shevlin, Claire Fyvie, Marylène Cloitre \& Thanos Karatzias}

To cite this article: Philip Hyland, Mark Shevlin, Claire Fyvie, Marylène Cloitre \& Thanos Karatzias (2019): The relationship between ICD-11 PTSD, complex PTSD and dissociative experiences, Journal of Trauma \& Dissociation, DOI: 10.1080/15299732.2019.1675113

To link to this article: https://doi.org/10.1080/15299732.2019.1675113

曲 Published online: 04 Oct 2019.

Submit your article to this journal $\longleftarrow$

Џlll Article views: 1

Q View related articles $₫$

View Crossmark data 


\title{
The relationship between ICD-11 PTSD, complex PTSD and dissociative experiences
}

\author{
Philip Hyland, PhD (10), Mark Shevlin, $\mathrm{PhD} \mathbb{1}^{\mathrm{b}}$, Claire Fyvie, $\mathrm{PhD}{ }^{\mathrm{c}}$, Marylène Cloitre, \\ $\mathrm{PhD}^{\mathrm{d}, \mathrm{e}}$, and Thanos Karatzias, PhD (1) ${ }^{\mathrm{c} f \mathrm{f}}$

\begin{abstract}
aDepartment of Psychology, Maynooth University, Kildare, Ireland; 'bSchool of Psychology, Ulster University, Derry, Northern Ireland; 'NHS Lothian, Rivers Centre for Traumatic Stress, Edinburgh, UK; ${ }^{d}$ National Center for PTSD Dissemination and Training Division, VA Palo Alto Health Care System, Palo Alto, CA, USA; 'Department of Psychiatry and Behavioural Sciences, Stanford University, Stanford, CA, USA; fSchool of Health \& Social Care, Edinburgh Napier University, Edinburgh, UK
\end{abstract}

\begin{abstract}
Debate exists in the trauma literature regarding the role of dissociation in traumatic stress disorders. With the release of the new ICD-11 diagnostic guideline for posttraumatic stress disorder (PTSD) and complex PTSD (CPTSD), this issue warrants further attention. In the current study, we provide a preliminary assessment of the associations between ICD-11 CPTSD and dissociative experiences. This study is based on a sample $(N=106)$ of highly traumatized clinical patients from the United Kingdom who completed measures of traumatic stress and dissociative experiences. The majority of participants met the diagnostic criteria for CPTSD $(69.1 \%, n=67)$, with few patients qualifying for a diagnosis of PTSD $(9.3 \%, n=9)$. Those with CPTSD had significantly higher levels of dissociative experiences compared to those with PTSD (Cohen's $d=1.04$ ) and those with no diagnosis (Cohen's $d=1.44$ ). Three CPTSD symptom clusters were multivariately associated with dissociation: Affective Dysregulation $(\beta=.33)$, Reexperiencing in the here and now $(\beta=.24)$, and Disturbed Relationships $(\beta=.22)$. These findings indicate that dissociative experiences are particularly relevant for clinical patients with CPTSD. Future longitudinal work will be needed to determine if dissociation is a risk factor for, or outcome of, CPTSD.
\end{abstract}

\section{ARTICLE HISTORY}

Received 16 December 2018

Accepted 9 June 2019

\section{KEYWORDS}

Posttraumatic stress disorder (PTSD); complex PTSD

(CPTSD); ICD-11; dissociation

Traumatic stress researchers have debated whether dissociation is dimensional or a taxon in trauma-related disorders (Brewin, 2003). Van der Hart, Nijenhuis, and Steele's $(2005,2006)$ structural theory of dissociation posits that individuals who have complex trauma reactions experience a division of their personality resulting in multiple dysfunctional outcomes such as fixation and avoidance. According to this theory, the severity of dissociation would be expected to increase from PTSD to Complex PTSD (CPTSD) to Dissociative Identity Disorder. Consistent with this expectation, there is evidence to suggest that higher levels of dissociation are evident in complex traumatic presentations compared to less complex presentations (Dorahy et al., 2013, 2015; 
Putnam et al., 1996). However, dissociation has also been suggested to reflect a unique cluster of PTSD symptoms that are only present in a small subset of trauma exposed individuals (Wolf et al., 2012, 2012); a view that led to the inclusion of a dissociative subtype of PTSD in the DSM-5 (APA, 2013). It has been difficult to resolve the debate regarding the association between dissociation and trauma because there is no universal agreement on what constitutes dissociation.

Two major types of dissociation have been described: Peritraumatic (or 'state') dissociation, which refers to dissociative experiences that occur during or in the immediate aftermath of a traumatic event; and persistent (or 'trait') dissociation which refers to chronic dissociation (Fleming \& Resick, 2016). Peritraumatic dissociation is relevant in complex and chronic traumatic presentations as it possible that these reactions are protective in the immediate aftermath of the traumatic event (Karatzias, Power, Brown, \& McGoldrick, 2010; Murphy, Elklit, Murphy, Hyland, \& Shevlin, 2017; Van der Kolk, 1987). However, it has also been argued that peritraumatic dissociation can interfere with normal traumatic processing, resulting in chronic traumatic stress responses (Ehlers \& Clark, 2000). Trait dissociation might also serve similar functions. Longitudinal data has found that persistent dissociation is a more important factor in the prediction of PTSD than peritraumatic dissociation (Briere, Scott, \& Weathers, 2005; Murray et al., 2002; Werner \& Griffin, 2012).

In an attempt to resolve this debate, Cardeña and Carlson (2011, p. 251) provided a broad definition of dissociation as "an experienced loss of information or control over mental processes that, under normal circumstances, are available to conscious awareness, self-attribution, or control". Carlson et al. (2018) operationalized this definition with the development of a self-report scale: The Dissociative Symptom Scale. Utilizing a heterogenous sample of clinical and non-clinical participants, four correlated factors of dissociative experiences were identified: (i) disconnectedness from one's sense of self and one's surroundings (labeled 'derealization/depersonalization'), (ii) cognitive-behavioral re-experiencing, (iii) gaps in memory and awareness, and (iv) sensory misperceptions.

Prior studies examining the relationship between dissociation and complex traumatic stress responses (Dorahy et al., 2013, 2015; van Dijke, Ford, Frank, \& Van der Hart, 2015) were limited by the fact that, until recently, CPTSD was never officially codified in any diagnostic nomenclature. This meant that different studies used different formulations of 'complex trauma'. The World Health Organization (WHO) added CPTSD to the $11^{\text {th }}$ version of the International Classification of Diseases under the category of 'Disorders specifically associated with stress' and thus provided the scientific community with a clear operational definition of CPTSD (WHO, 2018). Cloitre et al. (2018) developed and validated a self-report measure of CPTSD called the International Trauma Questionnaire (ITQ). This scale measures the six symptom clusters that characterize ICD-11 
CPTSD: re-experiencing of the trauma in the here and now, deliberate avoidance of internal and external traumatic reminders, a sense of current threat expressed as hypervigilance and hyperarousal (these three symptom clusters are shared with PTSD), emotional regulation difficulties such as hyper- or hypo-activation of emotions, a persistent negative view of the self, and interpersonal problems characterized by difficulties forming and maintaining relationships with others (these latter three symptom clusters are referred to as 'Disturbances in SelfOrganization' and distinguish CPTSD from PTSD in ICD-11). Considerable evidence has accrued in support of the ICD-11 model of CPTSD (see Brewin et al., 2017 for a review), however, there is limited data regarding the associations between ICD-11 CPTSD and dissociation.

The primary objective of this study was to explore the associations between ICD-11 CPTSD (at a diagnostic level and at a symptom cluster level) and dissociative experiences. In this study, we employed the model of dissociation operationalized by Carlson et al. (2018) and we examined the bivariate association between each CPTSD symptom cluster (re-experiencing, avoidance, sense of threat, affective dysregulation, negative self-concept, and disturbed relationships) and the total and subscale (derealization/depersonalization, cognitive-behavioral re-experiencing, gaps in memory and awareness, and sensory misperceptions) scores of dissociation. In addition to these exploratory analyzes, we formulated two hypotheses:

(1) Consistent with the structural theory of dissociation (Van Der Hart et al., 2005, 2006), we hypothesized that levels of dissociation would be highest for those meeting diagnostic criteria for CPTSD, followed by those meeting diagnostic criteria for PTSD, and finally, those without either diagnosis.

(2) Given the fact that the CPTSD symptom clusters of 'Re-experiencing in the here and now' and 'Affective Dysregulation' contain aspects of dissociative experiences (i.e., flashbacks and emotional numbing, respectively), we hypothesized that these symptom clusters would be associated with dissociation scores in a multivariate analysis.

\section{Methods}

\section{Participants and procedures}

Participants were attendees of a National Health Service trauma center in Scotland referred by general practitioners, psychiatrists, or psychologists for psychological therapy $(N=106)$. The relevant local ethics review board provided ethical approval, and all participants consented to their data being used for research purposes. The sample was primarily female $(93.4 \%)$ and of British origin (91.3\%) with a mean age of 39.25 years $(S D=10.94$, range $=19$ to 62). Most participants had finished post-secondary education (56.6\%), 
were currently unemployed (58.1\%), and single (59.2\%). All participants experienced a traumatic life event, measured using the Life Events Checklist for DSM-5 (Weathers et al., 2013). The mean number of traumatic life events was $6.99(S D=2.80)$ and the most commonly reported traumas were physical assault (95.1\%), sexual assault (83.5\%), and some other unwanted or uncomfortable sexual experience (82.5\%).

\section{Measures}

The International Trauma Questionnaire (ITQ; Cloitre et al., 2018) is a selfreport measure of the ICD-11 diagnoses of PTSD and CPTSD (accessible at www.traumameasuresglobal.com). The ITQ includes six items measuring the three PTSD symptom clusters (re-experiencing in the here and now, avoidance, and a sense of current threat) by two items each. Respondents are instructed to indicate how much they have been bothered by each symptom over the past month. Six items measure the three 'disturbances in selforganization' (DSO) symptom clusters that are unique to CPTSD (affective dysregulation, negative self-concept, and disturbed relationships), also measured by two items each. Respondents are instructed to answer the DSO questions in terms of how they typically feel, think about themselves, and relate to others. Three items measure functional impairment in the areas of social, work, and other important areas of life in relation to the PTSD and DSO symptoms, respectively. All ITQ items are answered using a five-point Likert scale ranging from 0 (Not at all) to 4 (Extremely). The psychometric properties of the ITQ have been demonstrated in multiple trauma-exposed samples (Cloitre et al., 2018; Hyland et al., 2017; Karatzias et al., 2016). The internal reliability of the PTSD $(\alpha=.74)$ and DSO $(\alpha=.81)$ items within the current sample were satisfactory.

Diagnosis of CPTSD is made if one symptom is present in the six clusters, and there is evidence of functional impairment associated with the PTSD and DSO symptoms. If the DSO symptom criteria are not met a PTSD diagnosis is made. The ICD-11 diagnostic rules only permit a diagnosis of PTSD or CPTSD, but not both. For diagnostic purposes, a symptom is deemed to be present based on a response of $\geq 2$ (Moderately) on the Likert-scale.

The 8-item Dissociative Symptoms Scale is a short version of the 20-item Dissociative Symptoms Scale (DSS: Carlson et al., 2018). This version of the DSS measures the four subscales (derealization/depersonalization, cognitivebehavioral re-experiencing, gaps in memory and awareness, and sensory misperceptions) by two items each. Individuals are asked to indicate how frequently they have experienced each dissociate experience over the past week on a fivepoint Likert scale ranging from 0 (Not at all) to 4 (More than once a day). Total scores range from $0-32$ and subscale scores range from $0-8$; in all cases higher scores reflect higher levels of dissociation. To test if the shortened version of the 
DSS measured the same four factors as the full scale, we performed a confirmatory factor analysis with robust maximum likelihood estimation in Mplus. The four-factor model was a very close fit to the data $\left(\chi^{2}(14)=8.79\right.$, $p=.844 ; \mathrm{CFI}=1.00 ; \mathrm{TLI}=1.05 ; \mathrm{RMSEA}=.00(90 \% \mathrm{CI}=.00, .06) ; \mathrm{SRMR}=.02)$; all standardized factor loadings were positive, statistically significant $(p s<.001)$ and ranged from .66 to .95; and all factors were all significantly $(p s<.05)$ and positively associated with one with $r$ s ranging from .29 (between cognitivebehavioral re-experiencing and sensory misperceptions) to .56 (between sensory misperceptions and gaps in memory and awareness). The internal reliability of the full scale in the current sample was good $(\alpha=.80)$.

\section{Data analysis}

Diagnostic rates for ICD-11 PTSD and CPTSD were calculated, and a oneway between-groups analysis of variance was used to compare total dissociation scores across the three diagnostic categories (1. No trauma diagnosis, 2. PTSD, and 3. CPTSD). Omega squared $\left(\omega^{2}\right)$, rather than eta squared, was used to calculate the overall effect size for the ANOVA test because eta squared can overestimate effect sizes when sample sizes are small. $\omega^{2}$ results can be interested in the same way as eta squared results where values $\leq .05$ indicate a small effect, values from .06 to .13 indicate a moderate effect, and values $\geq .14$ indicate a large effect (Cohen, 1988). The Tukey HSD test was used for post-hoc pairwise comparisons, and Cohen's $d$ (Cohen, 1988) was used to determine the magnitude of the difference in the means between each diagnostic group ( $d$ values $\leq .5=$ small effect, $.5-.8=$ moderate effect, $>$ .8 = large effect). Bivariate associations between the CPTSD symptom clusters and the total and subscale scores of dissociation were assessed using Pearson correlation coefficients. Finally, a standard multiple regression analysis was used to examine the multivariate associations between the six CPTSD symptom clusters and the total score of dissociation.

All analyzes were performed in SPSS version 25. There was minimal missing data ranging from $1.9 \%$ (sense of threat symptoms) to $6.6 \%$ (dissociation), and all missing data were handled using pairwise deletion in the different analyzes. Because five different correlation tests were planned, we applied a Bonferroni correction to control for an elevated type I error rate and thus set an alpha level of .01 for these tests. An a prior power analysis indicated a minimum required sample size of 111 for the one-way between groups ANOVA (assuming an alpha level of .05, an effect size of $f=.30$, and a power of .80); a minimum required sample size of 88 was for the two-tailed bivariate correlation analyzes (assuming an alpha level of .01, an effect size of $r=.35$, and a power of .80); a minimum required sample size of 52 for the standard multiple regression analysis (assuming an alpha level of .05, an effect size of $f=.30$, and a power of .80). 


\section{Results}

The majority of participants met the diagnostic criteria for CPTSD $(69.1 \%$, $n=67)$, a small percentage met the criteria for PTSD $(9.3 \%, n=9)$, and 21.6\% $(n=21)$ did not meet the diagnostic criteria for either PTSD or CPTSD. The descriptive statistics for the total and subscale scores of CPTSD and dissociation are presented in Table 1.

The overall and pairwise comparison results from the ANOVA test are presented in Table 2. Mean levels of dissociative experiences significantly differed across the diagnostic groups, and the magnitude of the difference was large. Those meeting the diagnostic criteria for CPTSD had significantly higher levels of dissociative experiences than those with PTSD $(d=1.04)$ and those with no diagnosis $(d=1.44)$. Those meeting the diagnostic criteria for PTSD had moderately higher levels of dissociative experiences than those with no diagnosis however this effect was not statistically significant $(d=0.65, p=.741)$.

The bivariate associations between the CPTSD symptom clusters and the dissociative experiences total and subscale scores are presented in Table 3. The total dissociative experiences score was significantly associated with each CPTSD symptom cluster and these correlations ranged from .27 (with Avoidance) to .57 (with Affective Dysregulation). The 'derealization/depersonalization' subscale was significantly associated with four of the six CPTSD

Table 1. Descriptive statistics for the ICD-11 complex PTSD symptom clusters and the total and subscale dissociation scores.

\begin{tabular}{lrrrcc}
\hline & Mean & 95\% Cls & Median & SD & Range \\
\hline PTSD: Re-experiencing in the here and now & 5.30 & $4.84,5.77$ & 6.00 & 2.35 & $0-8$ \\
PTSD: Avoidance of traumatic reminders & 5.88 & $5.55,6.21$ & 6.00 & 1.68 & $2-8$ \\
PTSD: Sense of threat & 6.02 & $5.61,6.43$ & 6.00 & 2.11 & $0-8$ \\
DSO: Affective dysregulation & 5.50 & $5.14,5.85$ & 6.00 & 1.84 & $1-8$ \\
DSO: Negative self-concept & 6.08 & $5.63,6.52$ & 7.00 & 2.28 & $0-8$ \\
DSO: Disturbed relationships & 6.12 & $5.76,6.48$ & 6.00 & 1.81 & $0-8$ \\
Total PTSD symptom score & 17.26 & $16.31,18.21$ & 18.00 & 4.76 & $3-24$ \\
Total CPTSD symptom score & 34.65 & $32.92,36.37$ & 36.50 & 8.52 & $9-48$ \\
Dissociation: Derealization/depersonalization & 2.91 & $2.41,3.41$ & 2.00 & 2.52 & $0-8$ \\
Dissociation: Cognitive-behavioral re-experiencing & 2.40 & $1.96,2.85$ & 2.00 & 2.25 & $0-8$ \\
Dissociation: Gaps in memory and awareness & 5.21 & $4.73,5.69$ & 5.00 & 2.41 & $0-8$ \\
Dissociation: Sensory misinterpretations & 1.48 & $1.12,1.85$ & 1.00 & 1.84 & $0-8$ \\
Total dissociation score & 12.01 & $10.74,13.28$ & 11.00 & 6.36 & $4-31$ \\
\hline
\end{tabular}

Table 2. One-way between groups analysis of variance results $(n=94)$.

\begin{tabular}{llcccccc}
\hline & & $\mathrm{N}$ & Mean & SD & F & P & $\omega 2$ \\
\hline Dissociation & No diagnosis & 19 & $6.63^{\mathrm{a}}$ & 2.19 & 12.95 & $<.001$ & .20 \\
& PTSD & 9 & $8.33^{\mathrm{a}}$ & 3.00 & & & \\
& CPTSD & 66 & $13.64^{\mathrm{a}}$ & 6.55 & & & \\
\hline
\end{tabular}

$\omega 2=$ Omega squared (values $\leq .05=$ small effect, values $.06-.13=$ moderate effect, values $\geq .14=$ large effect); ${ }^{a}$ pairwise analysis showed that dissociation levels were significantly higher for those with CPTSD than those with PTSD and those with no diagnosis. 
Table 3. Correlations between each CPTSD symptom cluster and the total and subscale dissociation scores.

\begin{tabular}{lccccc}
\hline & $\begin{array}{c}\text { Dissociation } \\
\text { Total }\end{array}$ & $\begin{array}{c}\text { Derealization/ } \\
\text { Depersonalization }\end{array}$ & $\begin{array}{c}\text { Cognitive-behavioral } \\
\text { re-experiencing }\end{array}$ & $\begin{array}{c}\text { Gaps in } \\
\text { memory \& } \\
\text { awareness }\end{array}$ & $\begin{array}{c}\text { Sensory } \\
\text { misperceptions }\end{array}$ \\
\hline PTSD: Re & $.51^{* *}$ & $.28^{*}$ & $.31^{*}$ & $.47^{* *}$ & $.39^{* *}$ \\
PTSD: Av & $.27^{*}$ & .15 & .17 & .24 & .21 \\
PTSD: Th & $.43^{*}$ & $.33^{*}$ & $.26^{*}$ & $.29^{*}$ & $.33^{*}$ \\
DSO: AD & $.57^{* *}$ & $.33^{*}$ & $.43^{* *}$ & $.45^{* *}$ & $.40^{* *}$ \\
DSO: NSC & $.38^{* *}$ & $.33^{*}$ & .24 & $.30^{*}$ & .23 \\
DSO: DR & $.52^{* *}$ & .24 & $.32^{*}$ & $.46^{* *}$ & $.35^{* *}$ \\
\hline
\end{tabular}

Statistical significance: ${ }^{*} p<.01 ;{ }^{* *} p<.001$; PTSD $=$ Posttraumatic stress disorder symptom cluster; $\mathrm{Re}=$ reexperiencing in the here and now; $A v=$ avoidance; Th = sense of threat; DSO = Disturbances in selforganization symptom cluster; $A D=$ affective dysregulation; $N S C=$ negative self-concept; $D R=$ disturbed relationships.

clusters and the correlations ranged from .15 to .33. The 'cognitivebehavioural re-experiencing' subscale was associated with four CPTSD clusters and the strongest association was with Affective Dysregulation $(r=.43)$. The 'gaps in memory and awareness' subscale was significantly associated with each CPTSD symptom cluster other than Avoidance, and the correlation ranged from .24 to .47. Finally, the 'sensory misperceptions' subscale was significantly associated with four CPTSD symptom clusters and the correlations ranged from .21 to .40 .

The six CPTSD symptom clusters explained $42.1 \%$ of variance in total dissociative experiences scores $\left(\operatorname{Adj} R^{2}=.421, F(6,89)=12.52, p<.001\right)$. Three symptom clusters remained significantly associated with dissociative experiences: Affective Dysregulation $(\beta=.33, p=.001)$, Re-experiencing in the here and now $(\beta=.24, p=.016)$, and Disturbed Relationships $(\beta=.22, p=.036)$.

\section{Discussion}

The purpose of the current study was to provide a preliminary assessment of the association between the recently published ICD-11 model of CPTSD and dissociative experiences. Our findings partially supported the study's first hypothesis as there was a pattern of increasing dissociative experiences scores from those with no diagnosis to those with PTSD to those with CPTSD; a finding consistent with the structural theory of dissociation (Van Der Hart et al., 2005, 2006). The magnitude of the difference in dissociative experiences scores between the CPTSD and PTSD diagnosis groups was substantial and this suggests that dissociation may be a clinically useful indicator of a differential diagnosis. The difference in dissociative experiences scores among patients with PTSD and those not meeting the criteria for a traumarelated diagnosis was of a moderate/clinically meaningful magnitude, however, the effect was not statistically significant. This null effect was almost certainly due to the low power of the test that resulted from a very small 
number of patients qualifying for a PTSD diagnosis, and consequently, this particular finding should be interpreted with great caution. Nonetheless, based on this small sample of trauma-exposed clinical patients, our findings suggest increasing levels of dissociative experiences as trauma responses become increasingly complex. This conclusion is consistent with empirical results obtained prior to the ICD-11 formulation of CPTSD (Dorahy et al., 2013, 2015; Putnam et al., 1996).

Dissociative experiences are embedded within the ICD-11 description of both PTSD and CPTSD. One of the three core symptom clusters of PTSD is 'Re-experiencing in the here and now' and the two constituent symptoms are nightmares of the traumatic event and flashbacks to the traumatic event where it feels as if the event is reoccurring in the present moment. Additionally, one of the core DSO symptom clusters (the symptom clusters that differentiate CPTSD from PTSD) is 'Affective Dysregulation', and one of its two constituent symptoms is a persistent feeling of emotional numbness. It was for this reason that we hypothesized that these symptom clusters would be most strongly associated with dissociative experiences scores in the multivariate analysis. We found that all CPTSD symptom clusters were positively correlated with dissociative experiences scores in a bivariate framework, however, the results of the multiple regression analysis showed that it was only 'Re-experiencing', 'Affective Dysregulation' and 'Disturbed Relationships' that were independently associated with dissociative experiences. Although we had not hypothesized a multivariate association between the 'Disturbed Relationships' symptom cluster and dissociation, this effect is not surprising given that dissociation is known to moderate distress by disrupting emotional relationships that activate traumatic material. LyonsRuth, Yellin, Melnick, and Atwood (2003) has argued that when a person is experiencing dissociative body detachment, this experience affects their ability to stay connected to people. Thus, dissociation can have an effect on one's ability to sustain close interpersonal relationships.

Considering the strength of the correlations between dissociative experiences and the different symptom clusters of CPTSD, our findings have important implications for the assessment and treatment of CPTSD. This is especially the case considering that symptoms of dissociation and PTSD appear to fluctuate concurrently together during treatment (Brand \& Stadnik, 2013; Lynch, Forman, Mendelsohn, \& Herman, 2008). In both studies, changes in dissociation were significantly related to changes in PTSD symptoms over the course of treatment. Thus, for the successful treatment of CPTSD it may be important to address dissociative experiences.

There are several limitations with the current study that must be acknowledged. The analyzes were based on cross-sectional data, therefore, no inferences of predictive relationships could be made. It will be important for future research to ascertain if persistent dissociative experiences precede, 
follow, or emerge concurrently with ICD-11 CPTSD symptoms. This study was also based on a small, predominately female, clinical sample with high rates of childhood trauma. The majority of the sample also qualified for a CPTSD diagnosis. The generalizability of the current findings to the wider trauma population is, therefore, substantially limited. Nonetheless, the current findings are valuable as they can be used to initiate further clinical research. Finally, the measure of dissociation used in the present study was an unvalidated short-form version of a relatively recently developed measure of dissociative experiences, as opposed to dissociative disorders. The internal reliability and factorial structure of this scale appeared to be sound within this sample but given its infrequent use the present results should be interpreted cautiously. Further work is required on the association between ICD11 PTSD/CPTSD and Dissociative Disorders.

Notwithstanding its limitations, this study provides valuable information regarding the association between ICD-11 CPTSD and dissociation. Further work is now required to extend our findings and explore the longitudinal associations between state and trait dissociation and CPTSD so that refinements can be made regarding how to most effectively conceptualize the role of dissociation in CPTSD, and consequently, how to integrate considerations of dissociation within the treatment of CPTSD.

\section{ORCID}

Philip Hyland (iD http://orcid.org/0000-0002-9574-7128

Mark Shevlin (1) http://orcid.org/0000-0001-6262-5223

Thanos Karatzias (D) http://orcid.org/0000-0002-3002-0630

\section{References}

American Psychiatric Association. (2013). Diagnostic and statistical manual of mental disorders (5th ed.). Washington, DC: Author.

Brand, B. L., \& Stadnik, R. (2013). What contributes to predicting change in the treatment of dissociation: Initial levels of dissociation, PTSD, or overall distress? Journal of Trauma \& Dissociation, 14, 328-341. doi:10.1080/15299732.2012.736929

Brewin, C. R. (2003). Current perspectives in psychology. Posttraumatic stress disorder: Malady or myth? New Haven, CT, US: Yale University Press.

Brewin, C. R., Cloitre, M., Hyland, P., Shevlin, M., Maercker, A., Bryant, R. A., ... Reed, G. M. (2017). A review of current evidence regarding the ICD-11 proposals for diagnosing PTSD and Complex PTSD. Clinical Psychology Review, 58, 1-15. doi:10.1016/j.cpr.2017.09.001

Briere, J., Scott, C., \& Weathers, F. (2005). Peritraumatic and persistent dissociation in the presumed etiology of PTSD. American Journal of Psychiatry, 162(12), 2295-2301. doi:10.1176/appi.ajp.162.12.2295 
Cardeña, E. B., \& Carlson, E. B. (2011). Acute stress disorder revisited. Annual Review of Clinical Psychology, 7, 245-267. doi:10.1146/annurev-clinpsy-032210-104502

Carlson, E. B., Waelde, L. C., Palmieri, P. A., Macia, K. S., Smith, S. R., \& McDade-Montez, E. (2018). Development and validation of the dissociative symptoms scale. Assessment, 25, 84-98. doi:10.1177/1073191116645904

Cloitre, M., Shevlin, M., Brewin, C. R., Bisson, J. I., Roberts, N. P., Maercker, A., \& Karatzias, T. (2018). The international trauma questionnaire: Development of a self-report measure of ICD-11 PTSD and complex PTSD. Acta Psychiatrica Scandinavica, 138, 536-546. doi:10.1111/acps.12956

Cohen, J. (1988). Statistical power analysis for the behavioral sciences (2nd ed.). Hillsdale, NJ: Lawrence Erlbaum Associates.

Dorahy, M. J., Corry, M., Shannon, M., Webb, K., McDermott, B., Ryan, M., \& Dyer, K. F. (2013). Complex trauma and intimate relationships: The impact of shame, guilt and dissociation. Journal of Affective Disorders, 147, 72-79. doi:10.1016/j.jad.2012.10.010

Dorahy, M. J., Middleton, W., Seager, L., McGurrin, P., Williams, M., \& Chambers, R. (2015). Dissociation, shame, complex PTSD, child maltreatment and intimate relationship self-concept in dissociative disorder, chronic PTSD and mixed psychiatric groups. Journal of Affective Disorders, 172, 195-203. doi:10.1016/j.jad.2014.10.008

Ehlers, A., \& Clark, D. M. (2000). A cognitive model of posttraumatic stress disorder. Behaviour Research and Therapy, 38(4), 319-345. doi:10.1016/S0005-7967(99)00123-0

Fleming, C. J. E., \& Resick, P. A. (2016). Predicting three types of dissociation in female survivors of intimate partner violence. Journal of Trauma and Dissociation, 17(3), 267-285. doi:10.1080/15299732.2015.1079807

Hyland, P., Shevlin, M., Brewin, C. R., Cloitre, M., Downes, A. J., Jumbe, S., ... Roberts, N. P. (2017). Factorial and discriminant validity of ICD-11 PTSD and CPTSD using the new international trauma questionnaire. Acta Psychiatrica Scandinavica, 136, 231-338. doi:10.1111/acps.12771

Karatzias, T., Power, K., Brown, K., \& McGoldrick, T. (2010). Posttraumatic symptomatology and dissociation in outpatients with chronic posttraumatic stress disorder. Journal of Trauma and Dissociation, 11, 83-92. doi:10.1080/15299730903143667

Karatzias, T., Shevlin, M., Fyvie, C., Hyland, P., Efthimiadou, E., Wilson, D., ... Cloitre, M. (2016). An initial psychometric assessment of an ICD-11 based measure of PTSD and complex PTSD (ICD-TQ): Evidence of construct validity. Journal of Anxiety Disorders, 44, 73-79. doi:10.1016/j.janxdis.2016.10.009

Lynch, S. M., Forman, E., Mendelsohn, M., \& Herman, J. (2008). Attending to dissociation: Assessing change in dissociation and predicting treatment outcome. Journal of Trauma and Dissociation, 9(3), 301-319. doi:10.1080/15299730802139063

Lyons-Ruth, K., Yellin, C., Melnick, S., \& Atwood, G. (2003). Childhood experiences of trauma and loss have different relations to maternal unresolved and hostile- helpless states of mind on the AAI. Attachment \& Human Development, 5(4), 330-352. doi:10.1080/ 14616730310001633410

Murphy, S., Elklit, A., Murphy, J., Hyland, P., \& Shevlin, M. (2017). A cross-lagged panel study of dissociation and posttraumatic stress in a treatment-seeking sample of survivors of childhood sexual abuse. Journal of Clinical Psychology, 73(10), 1370-1381. doi:10.1002/jclp.22439

Murray, J., Ehlers, A., Mayou, R. A., Murray, J., Ay, M., Ehlers, A., \& Mayou, R. A. (2002). Dissociation and post-traumatic stress disorder : Two prospective studies of road traffic accident survivors. The British Journal of Psychiatry, 180, 363-368. doi:10.1192/bjp.180.4.363

Putnam, F. W., Carlson, E. B., Ross, C. A., Anderson, G., Clark, P., Torem, M., ... Braun, B. G. (1996). Patterns of dissociation in clinical and nonclinical samples. Journal of Nervous and Mental Disease, 184(11), 673-679. doi:10.1097/00005053-199611000-00004 
Van Der Hart, O., Nijenhuis, E. R. S., \& Steele, K. (2005). Dissociation: An insufficiently recognized major feature of complex posttraumatic stress disorder. Journal of Traumatic Stress, 18(5), 413-423. doi:10.1002/jts.20049

Van Der Hart, O., Nijenhuis, E. R. S., \& Steele, K. (2006). The haunted self: Structural dissociation and the treatment of chronic traumatization. New York, NY: W.W. Norton.

Van der Kolk, B. A. (1987). Psychological Trauma. Washington, D.C.: American Psychiatric Press.

van Dijke, A., Ford, J. D., Frank, L. E., \& Van der Hart, O. (2015). Association of childhood complex trauma and dissociation with complex posttraumatic stress disorder symptoms in adulthood. Journal of Trauma \& Dissociation, 16, 428-441. doi:10.1080/15299732.2015.1016253

Weathers, F. W., Blake, D. D., Schnurr, P. P., Kaloupek, D. G., Marx, B. P., \& Keane, T. M. (2013). The life events checklist for DSM-5 (LEC-5). Instrument available from the National Center for PTSD at www.ptsd.va.gov

Werner, K. B., \& Griffin, M. G. (2012). Peritraumatic and persistent dissociation as predictors of PTSD symptoms in a female cohort. Journal of Traumatic Stress, 25, 401-407. doi:10.1002/jts.21725

Wolf, E. J., Lunney, C. A., Miller, M. W., Resick, P. A., Friedman, M. J., \& Schnurr, P. P. (2012). The dissociative subtype of PTSD: A replication and extension. Depression and Anxiety, 29, 679-688. doi:10.1002/da.21946

Wolf, E. J., Miller, M. W., Reardon, A. F., Ryabchenko, K. A., Castillo, D., \& Freund, R. (2012). A latent class analysis of dissociation and posttraumatic stress disorder: Evidence for a dissociative subtype. Archives of General Psychiatry, 69, 698-705. doi:10.1001/ archgenpsychiatry.2011.1574

World Health Organization. (2018). The ICD-11 for mortality and morbidity statistics. Retrieved from https://icd.who.int/browse11/1-m/en. 